\title{
Risco nutricional e cardiovascular em idosos quilombolas
}

\author{
Nutritional and cardiovascular disease risk in older persons living \\ in Quilombola communities
}

Thalita Costa da Silva (https://orcid.org/0000-0002-4458-5560) ${ }^{1}$

Carlos Martins Neto (https://orcid.org/0000-0002-6554-3087) ${ }^{1}$

Carolina Abreu de Carvalho (https://orcid.org/0000-0001-7900-4642) ${ }^{1}$

Poliana Cristina de Almeida Fonseca Viola (https://orcid.org/0000-0002-8875-5154) ${ }^{2}$

Lívia dos Santos Rodrigues (https://orcid.org/0000-0003-2933-6125) ${ }^{3}$

Bruno Luciano Carneiro Alves de Oliveira (https://orcid.org/0000-0001-8053-7972) ${ }^{1}$

\footnotetext{
${ }^{1}$ Programa de PósGraduação em Saúde

Coletiva, Universidade Federal do Maranhão. R. Barão de Itapary 155, Centro. 65020-070 São Luís MA Brasil. thalitacosta91@hotmail.com ${ }^{2}$ Departamento de Nutrição, Universidade Federal do Piauí. Teresina PI Brasil. ${ }^{3}$ Departamento de Puericultura e Pediatria, Faculdade de Medicina de Ribeirão Preto, Universidade de São Paulo. Ribeirão Preto SP Brasil.
}

\begin{abstract}
This article aims to assess nutritional and cardiovascular disease (CVD) risk based on anthropometric measures among older persons living in Quilombola communities in the state of Maranhão, Brazil. We conducted a cross-sectional study with 205 older persons living in 11 Quilombola communities in Bequimão, Maranhão. Nutritional and CVD risk were estimated according to sex and age group based on anthropometric indicators using Pearson's chi-square or Fisher's exact tests and analysis of variance, adopting a significance level of $p<0.05$. The study participants suffer precarious housing, basic sanitation and social conditions. Prevalence of nutritional and CDV risk was high across the sample, showing differences between sexes and age groups. Prevalence of excess weight was higher in women and the youngest age group, while prevalence of malnourishment and loss of muscle mass was higher in men and individuals aged 80 years and over. Prevalence of CVD risk was high across all age groups and higher in women than men. The older persons living in the Quilombola communities investigated by this study are socially vulnerable and showed high prevalence of low weight, loss of muscle mass and CDV risk. The prevalence of CVD risk was higher among women and the oldest age group.
\end{abstract}

Key words Older persons, Groups of African descent, Anthropometry, Nutrition, Cardiovascular
Resumo $O$ objetivo deste artigo é avaliar o risco nutricional e cardiovascular segundo medidas antropométricas em idosos quilombolas do estado do Maranhão. Trata-se de estudo transversal realizado em 11 comunidades remanescentes de quilombolas do município de Bequimão, Maranhão, Brasil. Realizou-se censo da população idosa que representou 205 pessoas. Foram estimados os riscos nutricional e cardiovascular por meio dos indicadores antropométricos segundo sexo e idade. Realizou-se Testes de Qui-quadrado de Pearson ou Exacto de Fisher e análises de variância. Diferenças foram consideradas estatisticamente significantes quando $p<0,05$. Idosos quilombolas vivem em precárias condições de moradia e de infraestrutura sanitária, com elevado risco nutricional e cardiovascular, mas com diferenças entre sexo e idade. O excesso de peso foi mais prevalente em mulheres e idosos mais jovens, enquanto os homens e idosos com 80 ou mais anos apresentaram-se mais desnutridos e com maior perda de massa corporal. O risco cardiovascular foi maior entre as mulheres e em todas as faixas etárias. Idosos quilombolas vivem em vulnerabilidade socioeconômica e apresentaram alta prevalência de baixo peso, perda de massa muscular e alto risco cardiovascular, sendo maior risco entre mulheres $e$ idosos do grupo de maior idade.

Palavras-chave Idosos, Grupos de ascendência africana, Antropometria, Nutrição, Cardiovascular 


\section{Introdução}

As comunidades quilombolas são territórios, predominantemente, rurais e localizadas em áreas remotas compostas em grande parte por descendentes de escravos fugidos que ainda vivem em vulneráveis condições de vida e saúde. Essas comunidades são encontradas em todas as regiões do Brasil, mas a grande maioria delas estão no Nordeste brasileiro, sobretudo Bahia (30,0\%) e Maranhão $(27,7 \%)^{1}$.

Essas comunidades representam espaços de resistência e luta por direitos e pela conservação de bens culturais e crenças religiosas, valores e práticas tradicionais enraizadas em sua ascendência africana. Quilombos foram alternativas de sobrevivência coletiva de escravos que se recusaram a aceitar regras impostas pelo sistema colonial brasileiro racista. Hoje eles constituem expressão da história de resistência à exclusão dos negros que adquiriram formas específicas de organização social, de relação com a terra e hábitos de vida e saúde ${ }^{1,2}$.

Nos últimos anos, aumentou o número de estudos epidemiológicos sobre a situação de saúde de quilombolas, mas estes ainda representam pequena fração dos estudos com população negra. As pesquisas disponíveis indicam precários níveis de saúde, pior qualidade de vida, de infraestrutura sanitária e de acesso a serviços sociais e saúde com impactos maiores entre os grupos dos extremos de idade, como idosos ${ }^{1}$ e crianças ${ }^{3}$. A importante variabilidade na situação de vida e saúde entre os grupos de quilombolas tem destacado as adicionais desigualdades que idosos experimentam nessas comunidades, que aumentam os seus riscos de adoecimento ${ }^{1,4}$.

O envelhecimento nos quilombos se caracteriza por ocorrer em situação de pobreza, elevadas necessidades de saúde, pior qualidade de vida, insegurança nutricional e múltiplo adoecimento crônico ${ }^{1}$. As desigualdades que enfrentam devemse à ampla exposição a privações socioeconômicas e materiais que estão historicamente submetidos desde o período escravagista. Esse acúmulo de desvantagens e desinvestimentos públicos ao longo de gerações e ciclos de vida refletem-se nos indicadores comumente utilizados para se avaliar o estado de saúde nutricional e seus riscos relacionados nos idosos, mas se mantendo diferenças entre seus subgrupos de sexo e idade ${ }^{5}$.

Os estudos que avaliam o estado nutricional e risco cardiovascular em quilombolas são escassos e, os poucos disponíveis com idosos mostram elevada prevalência de risco nutricional ${ }^{2,6-8}$. A maioria desses estudos fazem análises de específicos indicadores e não analisam em conjunto medidas distintas de avaliação antropométrica e risco cardiovascular, nem verificam se desvios nutricionais e risco cardiovascular diferem entre os grupos de sexo e idade, não permitindo saber o real risco que essas populações estão expostas.

Assim, este estudo buscou avaliar o risco nutricional e cardiovascular segundo as medidas antropométricas de idosos quilombolas do estado do Maranhão.

\section{Métodos}

\section{Área e população de estudo}

Este estudo faz parte do projeto Inquérito populacional sobre as Condições de Vida e Saúde dos Idosos Quilombolas de uma Cidade da Baixada Maranhense (Projeto IQUIBEQ). Trata-se de estudo transversal, de base domiciliar, realizado em onze comunidades remanescestes de quilombolas do município de Bequimão, Maranhão, Brasil. Neste município existiam 18 dessas comunidades, porém apenas onze eram oficialmente reconhecidas como remanescestes de quilombolas pela Fundação Cultural Palmares vinculada ao Ministério da Cultura, por isso estas foram selecionadas como locais deste estudo (Figura 1).

A amostra de estudo foi composta por idosos com 60 anos ou mais de idade, que foram recrutados por meio de articulação com a Secretaria de Assistência Social do município e Agentes Comunitários de Saúde (ACS) das comunidades. Os ACS realizaram levantamento prévio e construíram lista nominal com informações do sexo e data de nascimento, contabilizando 220 idosos. Todos os idosos foram convidados a participar da pesquisa, porém, após as recusas e dificuldades de encontrar os idosos nas comunidades em duas tentativas em datas distintas, a amostra final avaliada foi de 205 idosos.

A coleta de dados foi realizada durante a semana em horários diurnos entre os meses de julho a outubro de 2018. Previamente foi realizado um estudo-piloto para ajustes dos instrumentos e treinamento dos entrevistadores. Durante a coleta de dados os entrevistadores poderiam consultar ao manual de instruções para esclarecimento das dúvidas, além de estarem acompanhados dos pesquisadores responsáveis da pesquisa. 


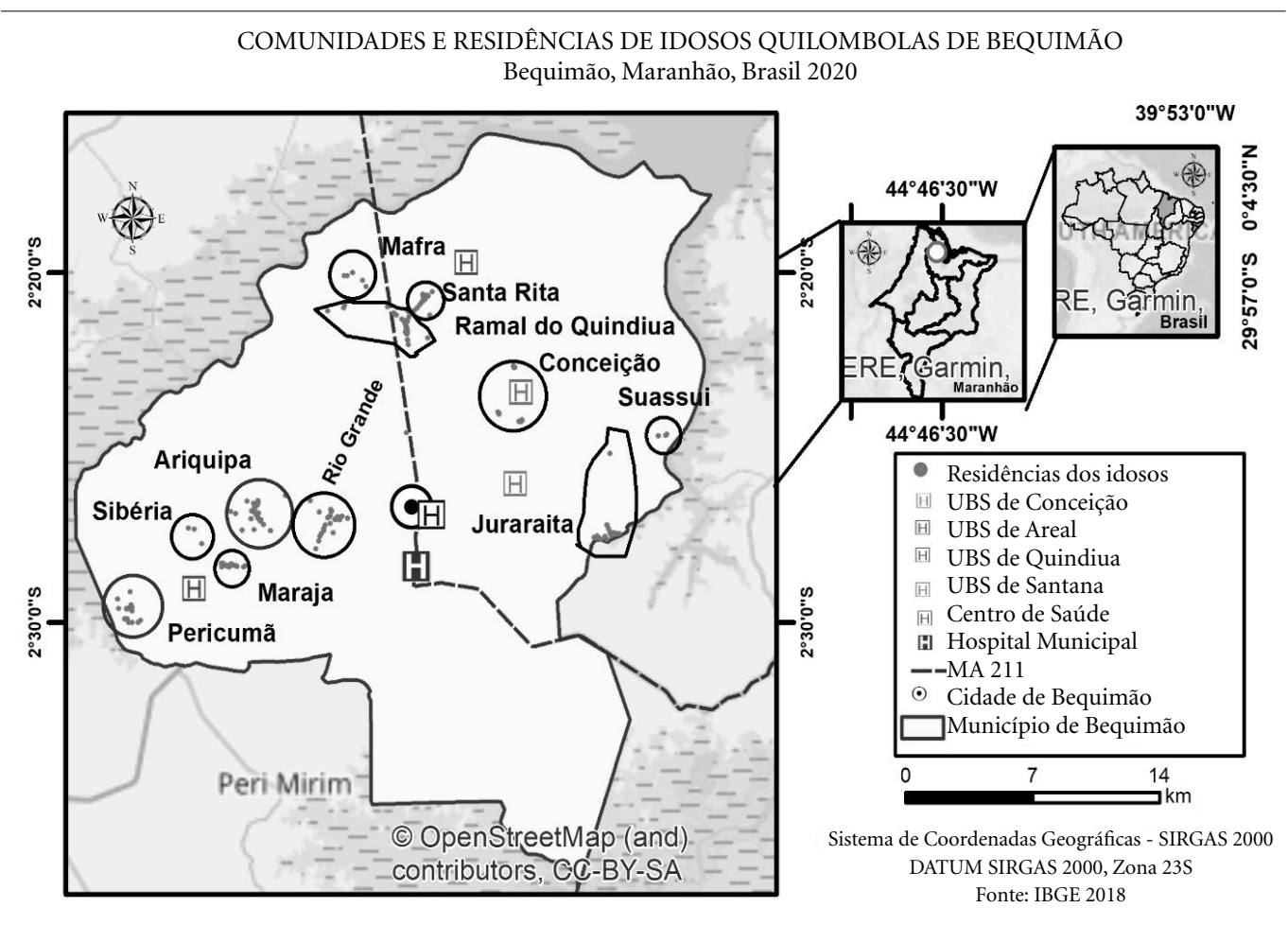

Figura 1. Localização geográfica das onze comunidades remanescentes de quilombolas em Bequimão (Projeto IQUIBEQ), Maranhão, Brasil, 2018.

Fonte: Autores.

\section{Questionários e variáveis do estudo}

Para este estudo foram utilizadas as variáveis socioeconômicas: sexo, idade, raça/cor da pele, situação conjugal, sabe ler e escrever, renda familiar em reais, estrato socioeconômico conforme Classe Social pelo Novo Critério Brasil ${ }^{9}$, recebimento de benefício do governo, número de cômodos por domicílio, material apropriado usado na construção das paredes, telhado e piso, abastecimento e tratamento de água, destino do esgoto e manejo de lixo.

Foram aferidas medidas antropométricas: Peso, Estatura, Perímetro da Cintura (PC), Perímetro do Quadril (PQ), Perímetro do Braço (PB) e Perímetro da Panturrilha Esquerda (PPE).

Para obtenção do peso corporal, os idosos foram pesados descalços e orientados a retirarem objetos e adornos que poderiam interferir no peso corporal real. Ao subir na plataforma da balança, o idoso deveria ficar ereto, com os pés juntos e os braços estendidos ao longo do cor$\mathrm{po}^{10}$. Foi utilizada uma balança digital eletrônica, da marca Omron ${ }^{\circledR}$, com capacidade de $200 \mathrm{~kg}$ e precisão de $100 \mathrm{~g}$.
A altura, em centímetros, foi aferida com estadiômetro portátil, marca Alturexata ${ }^{\circledR}$. O idoso foi posicionado com os pés paralelos e os calcanhares, panturrilhas, glúteos, costas e parte posterior da cabeça deveriam estar encostados no estadiômetro, ou no mínimo três deles ${ }^{10}$.

O PC foi aferido com o indivíduo de pé, ereto, com braços estendidos ao longo do corpo, sendo realizado no ponto médio entra a última costela e a crista ilíaca com uma fita métrica inextensível. Para o PQ, utilizou-se a fita inelástica, e o PQ foi medido na área de maior perímetro aparente da região glútea ${ }^{10}$.

A medida de PPE foi realizada com uma fita métrica inelástica, na sua parte mais protuberante, com o idoso sentado, a perna dobrada formando um ângulo de 90 graus com o joelho. Para o PB foi solicitado que o idoso flexionasse o cotovelo a 90 graus e com a fita métrica localizou-se o ponto médio do braço. Após relaxamento do braço, deixando-o livremente estendido ao longo do corpo, foi tomada a medida em cima do ponto marcado, sem fazer compressão ${ }^{11}$.

O IMC dos idosos foi classificado de acordo com os pontos de corte de Lipschitz ${ }^{10}$ em Baixo 
Peso (IMC $<22 \mathrm{~kg} / \mathrm{m}^{2}$ ); Eutrófico (IMC entre 22 $\mathrm{kg} / \mathrm{m}^{2}$ e $27 \mathrm{~kg} / \mathrm{m}^{2}$ ); e Excesso de Peso (que representa as pessoas com sobrepeso e obesidade) (IMC $>27 \mathrm{~kg} / \mathrm{m}^{2}$ ).

$\mathrm{O}$ perímetro do braço foi classificado de acordo com dados de referência do Third $\mathrm{Na}$ tional Health and Nutrition Examination Survey (NHANES III), utilizando-se como referência o percentil $50^{\circ}$ para população brasileira, com a seguinte equação: adequação da $P B(\%)=P B o b$ tida $(\mathrm{cm}) \times 100 / P B$ percentil $50^{\circ}$. Para a classificação do estado nutricional considerou-se: Desnutrição leve $<80 \%$; Eutrofia $90-110 \%$; Excesso de peso $110-120 \%$; Obesidade $>120 \%{ }^{12}$.

O risco cardiovascular foi avaliado com as medidas da PC e PQ. A razão cintura-quadril (RCQ) foi calculada usando a fórmula: $\mathrm{RCQ}=\mathrm{PC}(\mathrm{cm}) /$ PQ $(\mathrm{cm})$ e classificada de acordo com Lohman et al..$^{13}$ para indivíduos com mais de 60 anos, considerando para homens: Risco baixo $(<0,91)$, Moderado (entre 0,91-0,99) e Alto (1,00-1,03); e para mulheres: Risco baixo $(<0,76)$, Moderado (entre $0,76-0,84$ ) e Alto (entre $0,85-0,90)^{13}$. O PC foi classificado de acordo com os pontos de corte da $\mathrm{WHO}^{11}$ para adultos, sendo considerado risco: $\mathrm{PC}>94 \mathrm{~cm}$ para os homens e $>80 \mathrm{~cm}$ para as mulheres.

Para o PPE foi utilizada a classificação proposta pela $\mathrm{WHO}^{11}$, em que valores abaixo de 31 $\mathrm{cm}$ indicam perda de massa muscular. A PPE é bastante utilizada para avaliar a funcionalidade e quantidade do tecido muscular. Quanto maior seu valor melhor a capacidade funcional e menor a fragilidade do idoso ${ }^{14}$.

\section{Análise dos dados}

Foram calculadas as frequências absolutas e relativas para o conjunto de características socioeconômicas e demográficas dos idosos. As variáveis quantitativas (medidas antropométricas) foram também descritas por média, desvio-padrão, mediana e intervalo Interquartílico: percentil 25 e percentil 75. A distribuição dessas variáveis foi verificada por meio do teste Shapiro-Wilk. As medidas antropométricas Estatura, $\mathrm{PC}$ e $\mathrm{PB}$ apresentaram distribuição normal, e Peso, PQ e PPE sem distribuição normal. Em seguida, buscou-se verificar diferenças estatísticas nas estimativas descritivas (média e mediana) dessas medidas antropométricas segundo sexo e faixa etária ( 60 a 69,70 a 79 e $\geq 80$ anos). Para as comparações entre sexo, usou-se testes de Mann-Whitney (para diferenças entre as medianas) ou Teste $t$ para variâncias iguais (para diferenças entre as médias).
Por faixa etária, utilizou-se Kruskal-Wallis com comparações múltiplas (para diferenças entre as medianas) e ANOVA com comparações por Bonferroni (para diferenças entre as médias). Também se realizou, análise de correlação de Pearson entre Estatura, PC e PB e de Spearman entre Peso, PQ e PPE.

Foram estimados os riscos nutricional e cardiovascular por meio dos indicadores de IMC, PB, PPE, PC e RCQ segundo o sexo (masculino ou feminino) e os três grupos etários dos idosos. Testes de Qui-quadrado de Pearson ou Exacto de Fisher foram realizados para comparação de proporções.

Em todas as análises realizadas diferenças foram consideradas estatisticamente significantes quando $\mathrm{p}<0,05$. Todos os dados foram analisados no programa Stata ${ }^{\circledR}$ versão 14 (Stata Corp LP, College Station, Texas, Estados Unidos).

\section{Considerações éticas}

A pesquisa foi aprovada pelo Comitê de Ética em Pesquisa do Hospital Universitário da Universidade Federal do Maranhão (UFMA) (parecer favorável: 2.476 .488 de 28/01/2018) e todos os participantes assinaram o Termo de Consentimento Livre e Esclarecido antes da coleta.

\section{Resultado}

Foram avaliados 205 idosos, com mediana de idade de 70 anos (64-77). A maioria eram mulheres $(54,6 \%)$ e tinham entre 60 e 69 anos de idade $(49,3 \%)$. Consideravam-se de cor/raça preta $(58,5 \%)$, viviam sem cônjuge $(64,4 \%)$ e não sabiam ler e escrever (54,6\%). Apesar de 91,7\% ter a aposentadoria como fonte de renda e $63,9 \%$ ter renda familiar acima de um salário mínimo, $98,1 \%$ dos idosos pertenciam à classe econômica $\mathrm{D} / \mathrm{E}$. Quanto às condições de moradia, $68,8 \%$ residiam em casas com quatro a sete cômodos, apenas $27,8 \%$ tinham simultaneamente paredes, telhado e piso construídos com material adequado. A maioria não recebia abastecimento de água no domicílio, com $81,0 \%$ consumindo água de poço/nascente de dentro ou fora de sua propriedade. Sendo que $31,2 \%$ dos idosos não possuíam tratamento adequado da água no domicílio. Destino rudimentar ou a céu aberto do esgoto foi referido por $43,9 \%$ e nenhum deles tinha coleta regular de lixo dos domicílios (Tabela 1).

No total da população de estudo, observou-se pequena variabilidade das medidas antropométri- 
Tabela 1. Características socioeconômicas, demográficas e sanitárias de idosos quilombolas $\geq 60$ anos, Bequimão (Projeto IQUIBEQ), Maranhão, Brasil, 2018.

\begin{tabular}{|c|c|c|}
\hline Variáveis & $(\mathrm{N}=205)$ & $\%$ \\
\hline \multicolumn{3}{|l|}{ Sexo } \\
\hline Masculino & 93 & 45,4 \\
\hline Feminino & 112 & 54,6 \\
\hline \multicolumn{3}{|l|}{ Faixa etária (em anos) } \\
\hline 60 a 69 & 101 & 49,3 \\
\hline 70 a 79 & 65 & 31,7 \\
\hline$\geq 80$ & 39 & 19.0 \\
\hline \multicolumn{3}{|l|}{ Cor/raça } \\
\hline Preta & 120 & 58,5 \\
\hline Parda & 60 & 29,3 \\
\hline Outras & 25 & 12,2 \\
\hline \multicolumn{3}{|l|}{ Situação conjugal } \\
\hline Com cônjuge & 73 & 35,6 \\
\hline Sem cônjuge & 132 & 64,4 \\
\hline \multicolumn{3}{|l|}{ Sabe ler e escrever } \\
\hline Sim & 93 & 45,4 \\
\hline Não & 112 & 54,6 \\
\hline \multicolumn{3}{|c|}{ Renda familiar em salário mínimo de 954,00 (em reais) } \\
\hline$<1$ salário mínimo & 74 & 36,1 \\
\hline 1 a 2 salários mínimos & 131 & 63,9 \\
\hline \multicolumn{3}{|l|}{ Estrato socioeconômico* } \\
\hline $\mathrm{C}$ & 4 & 1,9 \\
\hline $\mathrm{D} / \mathrm{E}$ & 201 & 98,1 \\
\hline \multicolumn{3}{|l|}{ Benefícios recebidos } \\
\hline Aposentadoria/pensões & 188 & 91,7 \\
\hline Bolsa família & 14 & 8,3 \\
\hline \multicolumn{3}{|l|}{ Número de cômodos por domicílio } \\
\hline$\leq 3$ & 4 & 2,0 \\
\hline 4 a 7 & 141 & 68,8 \\
\hline$\geq 8$ & 60 & 29,3 \\
\hline \multicolumn{3}{|c|}{$\begin{array}{l}\text { Material predominante utilizado na construção de paredes, } \\
\text { telhado e piso é simultaneamente adequado }\end{array}$} \\
\hline Sim & 57 & 27,8 \\
\hline Não & 148 & 72,2 \\
\hline \multicolumn{3}{|l|}{ Abastecimento de água } \\
\hline Rede geral & 37 & 18,0 \\
\hline Poço ou nascente na propriedade & 123 & 60,0 \\
\hline Poço ou nascente na fora propriedade & 43 & 21,0 \\
\hline Outras formas & 2 & 1,0 \\
\hline \multicolumn{3}{|l|}{ Tratamento de água no domicílio } \\
\hline Apropriado & 141 & 68,8 \\
\hline Não apropriado & 64 & 31,2 \\
\hline \multicolumn{3}{|c|}{ Destino do esgoto de banheiros/sanitários do domicílio } \\
\hline Fossa séptica & 115 & 56,1 \\
\hline Fossa rudimentar/céu aberto & 90 & 43,9 \\
\hline \multicolumn{3}{|l|}{ Manejo do lixo } \\
\hline Jogado a céu aberto & 22 & 10,7 \\
\hline Queimado/enterrado & 183 & 89,3 \\
\hline
\end{tabular}

${ }^{\star}$ Não houve idosos no estrato social A e B.

Fonte: Autores. 
cas entre os 205 idosos avaliados. Contudo, ao se estratificar por sexo e idade diferenças apareceram. Segundo o sexo, ocorreram diferenças estatisticamente significantes nas medidas de peso, estatura, PC, PQ e PPE. Homens apresentaram maiores valores de peso $(p=0,001)$, estatura $(p=0,001)$ e PPE $(\mathrm{p}=0,002)$ e as mulheres de PC $(\mathrm{p}=0,049)$ e $P Q(p=0,006)$. Entre os grupos etários, observouse diferenças estatisticamente significantes nas medidas de peso $(\mathrm{p}=0,002), \mathrm{PB}(\mathrm{p}=0,026)$ e PPE $(\mathrm{p}=0,001)$. Os idosos com $\geq 80$ anos de idade apresentaram os piores valores das medidas avaliadas. Quando comparados aos idosos entre 60 e 69 anos, apresentaram valores estatisticamente significantes menor de peso $(p=0,001), P B(p=0,007) \mathrm{e}$ $\operatorname{PPE}(\mathrm{p}=0,001)$. Já em relação aos de 70 a 79 anos os menores valores ocorrem para peso $(\mathrm{p}=0,009)$ e PPE ( $\mathrm{p}=0,023)$ (Tabela 2).

Observou-se correlação estatisticamente significante $(\mathrm{p}=0,001)$ entre as seguintes medidas:

Tabela 2. Medidas descritivas de variáveis antropométricas de idosos quilombolas $\geq 60$ anos segundo sexo e idade, Bequimão (Projeto IQUIBEQ), Maranhão, Brasil, 2018.

\begin{tabular}{|c|c|c|c|c|c|c|c|c|}
\hline \multicolumn{2}{|c|}{ Variáveis } & $\begin{array}{l}\text { Medidas } \\
\text { Descritivas }\end{array}$ & $\begin{array}{l}\text { Peso } \\
(\mathrm{Kg})\end{array}$ & $\begin{array}{l}\text { Estatura } \\
(\mathrm{cm})\end{array}$ & $\begin{array}{l}\mathrm{PC} \\
(\mathrm{cm})\end{array}$ & $\begin{array}{l}P Q \\
(\mathrm{~cm})\end{array}$ & $\begin{array}{l}\mathrm{PB} \\
(\mathrm{cm})\end{array}$ & $\begin{array}{l}\text { PPE } \\
(\mathrm{cm})\end{array}$ \\
\hline \multirow{5}{*}{\multicolumn{2}{|c|}{ Total }} & Média & 57,9 & 1,53 & 87,5 & 94,5 & 28,2 & 31,6 \\
\hline & & $\mathrm{DP}$ & 9,8 & 0,08 & 9,7 & 7,4 & 3,2 & 3,4 \\
\hline & & P25 & 51,2 & 1,48 & 80,0 & 90,0 & 26,0 & 30,0 \\
\hline & & Mediana & 56,7 & 1,53 & 87,0 & 93,0 & 28,0 & 32,0 \\
\hline & & P75 & 63,3 & 1,6 & 94,0 & 99,0 & 30,0 & 33,5 \\
\hline \multirow[t]{10}{*}{ Sexo } & Masculino & Média & 60,5 & $1,59^{1}$ & $86,1^{1}$ & 92,6 & $28,2^{1}$ & 32,1 \\
\hline & & $\mathrm{DP}$ & 9,5 & 0,07 & 9,3 & 6,3 & 3,2 & 3,5 \\
\hline & & P25 & 53,6 & 1,54 & 79,0 & 89,0 & 26,5 & 31,0 \\
\hline & & Mediana & $60,0^{2}$ & 1,60 & 85,3 & $93,0^{2}$ & 28,0 & $32,5^{2}$ \\
\hline & & P75 & 65,8 & 1,64 & 93,5 & 96,0 & 30,0 & 34,0 \\
\hline & Feminino & Média & 55,9 & $1,49^{1}$ & $88,8^{1}$ & 96,1 & $28,2^{1}$ & 31,1 \\
\hline & & DP & 9,6 & 0,06 & 9,9 & 8,0 & 3,2 & 3,2 \\
\hline & & P25 & 49,1 & 1,45 & 83,0 & 91,0 & 26,0 & 29,0 \\
\hline & & Mediana & $54,4^{2}$ & 1,49 & 89,0 & $94,0^{2}$ & 28,0 & $31,0^{2}$ \\
\hline & & P75 & 60,6 & 1,53 & 96,0 & 101,0 & 30,0 & 32,0 \\
\hline p-valor & & & $0,001^{2}$ & $0,001^{1}$ & $0,049^{1}$ & $0,006^{2}$ & $0,971^{1}$ & $0,002^{2}$ \\
\hline \multirow{15}{*}{$\begin{array}{l}\text { Faixa etária } \\
\text { (em anos) }\end{array}$} & 60 a 69 & Média & 59,3 & $1,54^{3}$ & $87,0^{3}$ & 94,5 & 28,6 & 32,1 \\
\hline & & DP & 9,9 & 0,08 & 9,4 & 7,2 & 2,9 & 3,6 \\
\hline & & P25 & 53,5 & 1,49 & 80,0 & 91,0 & 27,0 & 31,0 \\
\hline & & Mediana & $57,9^{4 \star}$ & 1,55 & 86,0 & $93,5^{4}$ & $28,8^{4 * \star}$ & $32,0^{4 * * *}$ \\
\hline & & P75 & 65,0 & 1,61 & 93,0 & 97,0 & 30,0 & 34,0 \\
\hline & 70 a 79 & Média & 58,3 & $1,53^{3}$ & $89,1^{3}$ & 95,8 & 28,2 & 31,5 \\
\hline & & $\mathrm{DP}$ & 9,7 & 0,07 & 9,5 & 7,3 & 3,4 & 2,9 \\
\hline & & P25 & 50,8 & 1,48 & 81,0 & 90,0 & 26,0 & 29,5 \\
\hline & & Mediana & $57,5^{4 \$}$ & 1,53 & 88,0 & $95,0^{4}$ & $28,0^{4}$ & $32,0^{4 \$ \$}$ \\
\hline & & P75 & 63,1 & 1,59 & 96,5 & 100,0 & 31,0 & 33,0 \\
\hline & 80 ou mais & Média & 53,7 & $1,52^{3}$ & $86,4^{3}$ & 92,6 & 27,0 & 30,2 \\
\hline & & $\mathrm{DP}$ & 8,7 & 0,09 & 10,7 & 8,0 & 3,3 & 3,1 \\
\hline & & P25 & 47,7 & 1,47 & 79,0 & 87,0 & 25,0 & 28,0 \\
\hline & & Mediana & $51,6^{4 * \$}$ & 1,52 & 86,0 & $91,0^{4}$ & $26,0^{4 * *}$ & $30,0^{4^{* * *} \$ \$}$ \\
\hline & & P75 & 57,3 & 1,57 & 95,0 & 98,0 & 30,0 & 32,5 \\
\hline p-valor & & & $0,002^{4}$ & $0,117^{3}$ & $0,291^{3}$ & $0,052^{4}$ & $0,026^{4}$ & $0,001^{4}$ \\
\hline
\end{tabular}

Fonte: Autores. 
peso e PQ $(r=0,74)$, peso e PPE $(r=0,74)$, PQ e PPE $(r=0,59)$, e entre PC e PB $(r=0,68)$ (dados não mostrados).

O cálculo do IMC indicou que 52,7\% dos idosos estavam eutróficos, 25,9\% tinham baixo peso e $21,4 \%$ em excesso de peso. Embora as mulheres tivessem maior prevalência de excesso de peso e menor de baixo peso não houve diferença significante em relação aos homens $(\mathrm{p}=0,188)$. Quando observa-se a $\mathrm{PB}, 51,7 \%$ apresentaram desnutrição. A proporção de desnutrição foi maior entre homens e a de excesso de peso maior nas mulheres, mas sem diferença estatística significante entre eles $(p=0,174)$. Segundo a PPE mais de um terço $(34,0 \%)$ dos idosos foram avaliados como desnutridos, sendo estatisticamente $(\mathrm{p}=0,01)$ maior a prevalência nas mulheres $(41,8 \%)$ do que nos homens $(24,4 \%)$ (Tabela 3 ).

Quanto ao risco cardiovascular avaliado pelo PC, 54,1\% dos idosos apresentaram-se em risco, sendo significantemente $(p=0,001)$ maior esse risco nas mulheres (79,3\%). Já segundo a RCQ, apenas $19,2 \%$ estavam em baixo risco e $52,7 \%$ em risco elevado. As mulheres apresentaram maior prevalência de risco elevado de acordo com a RCQ do que homens ( $\mathrm{p}=0,001)$ (Tabela 3 ).
O baixo peso foi maior entre idosos $\geq 80$ anos $(43,6 \%)$ e o excesso de peso entre os de 60 a 69 anos $(22,8 \%)$ e 70 a 79 anos $(24,6 \%)$, mas sem diferenças estatísticas. A medida de PB indicou que quanto maior a idade, menor a prevalência de desnutrição (de 59,0\% até 69 anos para $43,6 \%$ em $\geq 80$ anos), porém maior a de excesso de peso (de $8,0 \%$ para 28,2\% nos extremos de idade; $\mathrm{p}=0,031)$. $\mathrm{O}$ PPE indicou que a prevalência de desnutrição foi maior nos idosos $\geq 80$ anos $(59,0 \%)$ do que nos de 60 a 69 anos $(24,5 \%)(\mathrm{p}=0,001)$. Para os demais indicadores não houve diferenças estatisticamente significantes (Tabela 4).

\section{Discussão}

Este estudo indicou que os idosos quilombolas vivem em precárias condições de moradia e de infraestrutura sanitária e social, com elevado risco nutricional e cardiovascular, mas com diferenças importantes segundo o sexo e idade.

Essas condições socioeconômicas e sanitárias desfavoráveis são consistentes com outros estudos realizados em comunidades quilombolas ${ }^{15,16}$. Os processos históricos de segregação e discrimi-

Tabela 3. Padrão antropométrico segundo o sexo de idosos quilombolas $\geq 60$ anos, Bequimão (Projeto IQUIBEQ), Maranhão, Brasil, 2018.

\begin{tabular}{|c|c|c|c|c|c|c|c|}
\hline \multirow{2}{*}{ Medidas } & \multicolumn{2}{|c|}{ Total } & \multicolumn{2}{|c|}{ Masculino } & \multicolumn{2}{|c|}{ Feminino } & \multirow{2}{*}{ p-valor } \\
\hline & $\mathbf{n}$ & $\%$ & $\mathbf{n}$ & $\%$ & $\mathbf{n}$ & $\%$ & \\
\hline \multicolumn{8}{|l|}{ IMC } \\
\hline Baixo peso & 53 & 25,9 & 29 & 31,2 & 24 & 21,4 & 0,188 \\
\hline Normal & 108 & 52,7 & 48 & 51,6 & 60 & 53,6 & \\
\hline Excesso de peso & 44 & 21,4 & 16 & 17,2 & 28 & 25,0 & \\
\hline \multicolumn{8}{|l|}{ Perímetro do Braço (PB) } \\
\hline Desnutrição & 105 & 51,7 & 55 & 59,8 & 50 & 45,1 & 0,174 \\
\hline Eutrofia & 61 & 30,1 & 23 & 25,0 & 38 & 34,2 & \\
\hline Excesso de peso & 36 & 17,7 & 14 & 15,2 & 22 & 19,8 & \\
\hline Obesidade & 1 & 0,5 & 0 & 0 & 1 & 0,9 & \\
\hline \multicolumn{8}{|c|}{ Perímetro da Panturrilha Esquerda (PPE) } \\
\hline Desnutrição & 68 & 34,0 & 22 & 24,4 & 46 & 41,8 & 0,010 \\
\hline Eutrofia & 132 & 66,0 & 68 & 75,6 & 64 & 58,2 & \\
\hline \multicolumn{8}{|l|}{ Perímetro da Cintura (PC) } \\
\hline Sem risco & 92 & 44,9 & 69 & 75,0 & 23 & 20,7 & 0,001 \\
\hline Risco & 111 & 54,1 & 23 & 25,0 & 88 & 79,3 & \\
\hline \multicolumn{8}{|c|}{ Razão cintura quadril (RCQ: PC/PQ) } \\
\hline Risco baixo & 39 & 19,2 & 38 & 41,3 & 1 & 0,9 & 0,001 \\
\hline Risco moderado & 57 & 28,1 & 42 & 45,7 & 15 & 13,5 & \\
\hline Risco alto & 107 & 52,7 & 12 & 13,0 & 95 & 75,6 & \\
\hline
\end{tabular}

${ }^{1}$ Teste qui-quadrado de Pearson ou Exato de Pearson. 


\begin{tabular}{|c|c|c|c|c|c|c|c|c|c|c|}
\hline \multicolumn{11}{|c|}{ faixa etária (em anos) de idosos quilombolas $\geq 60$ anos, Bequimão } \\
\hline & \multirow{2}{*}{ Medidas } & \multicolumn{2}{|c|}{ Total } & \multicolumn{2}{|c|}{60 a 69} & \multicolumn{2}{|c|}{70 a 79} & \multicolumn{2}{|c|}{80 ou mais } & \multirow{2}{*}{ p-valor ${ }^{1}$} \\
\hline & & $\mathrm{n}$ & $\%$ & $\mathbf{n}$ & $\%$ & $\mathrm{n}$ & $\%$ & $\mathbf{n}$ & $\%$ & \\
\hline & \multicolumn{10}{|l|}{ IMC } \\
\hline & Baixo peso & 53 & 25,9 & 22 & 21,8 & 14 & 21,5 & 17 & 43,6 & 0,079 \\
\hline & Normal & 108 & 52,7 & 56 & 55,4 & 35 & 53,9 & 17 & 43,6 & \\
\hline & Excesso de peso & 44 & 21,4 & 23 & 22,8 & 16 & 24,6 & 5 & 12,8 & \\
\hline & \multicolumn{10}{|l|}{ Perímetro do Braço (PB) } \\
\hline & Desnutrição & 105 & 51,7 & 59 & 59,0 & 29 & 45,3 & 17 & 43,6 & 0,031 \\
\hline & Eutrofia & 61 & 30,1 & 32 & 32,0 & 19 & 28,1 & 11 & 28,2 & \\
\hline & Excesso de peso & 36 & 17,7 & 8 & 8,0 & 17 & 26,6 & 11 & 28,2 & \\
\hline & Obesidade & 1 & 0,5 & 1 & 1,0 & 0 & 0,0 & 0 & 0,0 & \\
\hline & \multicolumn{10}{|c|}{ Perímetro da Panturrilha Esquerda (PPE) } \\
\hline & Eutrofia & 132 & 66,0 & 74 & 75,5 & 42 & 66,7 & 16 & 41,0 & 0,001 \\
\hline & Desnutrição & 68 & 34,0 & 24 & 24,5 & 21 & 33,3 & 23 & 59,0 & \\
\hline & \multicolumn{10}{|l|}{ Perímetro da Cintura (PC) } \\
\hline & Sem risco & 92 & 44,9 & 54 & 53,5 & 24 & 38,1 & 14 & 35,9 & 0,066 \\
\hline & Risco & 111 & 54,1 & 47 & 46,5 & 39 & 61,9 & 25 & 61,1 & \\
\hline & \multicolumn{10}{|c|}{ Razão cintura quadril (RCQ: PC/PQ) } \\
\hline & Risco baixo & 39 & 19,2 & 23 & 22,8 & 11 & 17,5 & 5 & 12,8 & 0,239 \\
\hline & Risco moderado & 57 & 28,1 & 32 & 31,7 & 13 & 20,6 & 12 & 30,8 & \\
\hline & Risco alto & 107 & 52,7 & 46 & 45,5 & 39 & 61,9 & 22 & 56,4 & \\
\hline
\end{tabular}

${ }^{1}$ Teste qui-quadrado de Pearson ou Exato de Pearson.

Fonte: Autores.

nação racial a que essas comunidades foram submetidas fizeram com que nelas fossem acumuladas desvantagens ao longo de gerações, expondo pessoas negras à exclusão e marginalização. Essa condição gerou múltiplas barreiras práticas de acesso aos serviços sociais e de saúde, que impactaram negativamente no bem-estar e saúde dos quilombolas. Essas desigualdades de longo prazo continuam sendo capturadas pelos indicadores sociais e de saúde entre idosos quilombolas, especialmente em realidades mais empobrecidas tais como no estado do Maranhão ${ }^{1}$. Com isso, as históricas desigualdades impostas às comunidades quilombolas também geram marcas na saúde do idoso ${ }^{17}$, visto que a população idosa cresce no Brasil, mas com elevada necessidade de saúde e em sobreposição de riscos socioeconômicos, sanitários, nutricionais e cardiovasculares ${ }^{1,18}$.

A maioria dos idosos apresentou IMC adequado. Porém, quando estratificados por sexo, as mulheres apresentavam mais excesso de peso que homens, como evidenciado em estudo realizado no município do Paraná e em Vitória da Conquista, Bahia ${ }^{6}$. Contudo, outras pesquisas mostraram que entre idosos, homens tinham prevalência de sobrepeso/obesidade muito superior às mulheres ${ }^{19}$. Essas divergências encontradas na literatura podem indicar que a situação de má nutrição pode estar associada a características individuais e assim depender da população analisada ${ }^{20}$.

Quanto ao peso, observou-se que as medidas de tendência central diminuíram quando a faixa etária aumentava e se associaram ao sexo feminino. Estes achados são corroborados por estudo realizado com idosos em sete cidades brasileiras. Os estudos verificaram que a prevalência de baixo peso nessa população era de $12,0 \%$, sendo mais elevada nos idosos de 80 anos ou mais ${ }^{21}$ e que o sexo feminino, autoavaliação negativa de saúde e aumento da pressão arterial média estavam associados à elevação do $\mathrm{IMC}^{22}$. Segundo Venturini et $a l .^{23}$ as mulheres apresentam mais excesso de peso, devido ao acúmulo maior de gordura visceral e subcutânea do que os homens. Além disso, desordens hormonais decorrentes da menopausa podem levar ao aumento de peso e adiposidade ${ }^{23}$.

Outra possível explicação seria o diferencial de gasto energético por sexo associado ao tipo mais frequente de atividade laboral realizada por 
homens e mulheres ${ }^{24}$. Por residirem em comunidades rurais, homens tendem a exercer mais atividades agrícolas baseadas na lavoura e pecuária de animais de grande porte em propriedades longe dos domicílios. Enquanto as mulheres, dedicam-se mais aos afazeres domésticos, criação de animais de pequeno porte e cultivo de hortaliças na área peridomiciliar ${ }^{25}$. Tais diferenças podem gerar excedentes de gordura corporal.

No que diz respeito à potencial relação entre consumo alimentar e estado nutricional, é possível que os resultados aqui observados possam sofrer influência da baixa qualidade da dieta, embora no presente estudo não tenha sido avaliado. Estudos com comunidades quilombolas têm reportado o baixo consumo de frutas e verduras nestas populações, o que contribui para aumento do peso e do risco cardiovascular ${ }^{2,26}$. Em pesquisa realizada em comunidade quilombola em Belém-PA, observou-se que apesar da variedade de frutas e hortaliças plantados na comunidade, o consumo por eles era baixo, pois a maior parte desses alimentos eram vendidos. Além disso, o consumo desses alimentos naturais era substituído por produtos industrializados, os quais favorecem o excesso de peso devido ao seu alto valor calórico ${ }^{27}$.

Neste estudo, apesar de não haver diferença da média de PB entre os sexos, como observado também por Silva et al..$^{19}$, evidenciou-se pelo PB maiores prevalências de desnutrição entre os idosos avaliados. Resultado semelhante foi encontrado por Silva et al. ${ }^{28}$ no Hospital Universitário do Pará, onde foi observada média mais baixa do PB em idosos com desnutrição (22,26 $\mathrm{cm})$ do que com aqueles com risco de desnutrição $(25,98 \mathrm{~cm})^{28}$. Dentre os parâmetros antropométricos avaliados em estudo realizado no Hospital Universitário do Recife-PE, o PB foi o que se mostrou mais eficaz na identificação de déficit nutricional $^{29}$.

Observou-se segundo o PPE que 34\% dos idosos estavam desnutridos, e considerando a faixa etária e sexo, maiores prevalências de desnutrição foram encontradas entre os idosos com mais de 80 anos e entre as mulheres. Outros estudos que avaliaram o estado nutricional em idosos verificaram prevalências de desnutrição inferior a esta pesquisa, variando de $2,2 \%$ a $7,9 \%{ }^{30}$, possivelmente esses resultados diversos se devem pelas diferenças socioeconômicas entre as populações estudadas. Achados semelhantes foram apresentados por Miranda et al. ${ }^{31}$ com idosos em Benevides-PA, que observaram que a desnutrição aumentou com a idade assim como Almeida et al. ${ }^{32}$, ao avaliar indicadores de saúde de quilombolas de Salvaterra-PA, classificaram 19,1\% como desnutridos segundo PPE. Como este indicador é também utilizado para avaliação de sarcopenia ${ }^{33}$, condição caracterizada pela perda generalizada e progressiva da força e massa muscular esquelética que ocorre como um processo natural do envelhecimento ${ }^{34}$, faz-se necessário o monitoramento desta medida uma vez que esta perda traz prejuízos para a funcionalidade e consequentemente para a qualidade de vida e bem-estar dos idosos.

Em relação ao risco cardiovascular, ao analisar a RCQ, observou-se que as mulheres estavam em maior risco, principalmente aquelas com mais de 70 anos, bem como outros estudos demonstraram ${ }^{35,36}$. As mulheres também apresentavam maior média do PC e percentual de risco três vezes maior que nos homens neste estudo, como também foi evidenciado em pesquisa desenvolvida no Amazonas, na população quilombola, onde mulheres também tinham média de PC mais elevados que homens, com diferenças estatisticamente significantes ${ }^{19}$. Em outro estudo, realizado com idosos do município de Es-

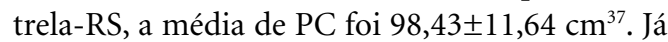
Cordovil e Almeida ${ }^{38}$, não encontraram diferença nas médias do PC entre homens e mulheres quilombolas de Salvaterra-PA, embora tenham verificado uma forte correlação entre essa variável e IMC, demonstrando o risco nutricional da população estudada. Barbosa et al. ${ }^{39}$, apesar de mostrarem uma alta prevalência de hipertensão e dislipidemia em uma comunidade quilombola do Maranhão, também observaram que outros fatores de risco independentes para eventos cardiovasculares foram baixos ${ }^{39}$.

Entre grupos raciais e étnicos minoritários de outros países, com semelhante ancestralidade africana como os remanescentes de quilombos deste estudo, existem desigualdades bem estabelecidas nos resultados de saúde cardiovascular e nutricio$\mathrm{mal}^{40-42}$. Os afro-americanos têm carga maior de doenças e eventos cardiovasculares. Estes também apresentam prevalência mais alta de fatores de risco, que não são reconhecidos e, portanto, não são tratados, o que coloca esses indivíduos em maior probabilidade de sofrer resultados adversos e, assim, morbimortalidade potencialmente mais alta. Inclusive quando idosos, a prevalência de fatores de risco cardiovascular é ainda maior ${ }^{40}$. Em 2013, as doenças cardiovasculares causaram quase um milhão de mortes na África Subsaariana, constituindo 38,3\% das mortes por doenças não transmissíveis ${ }^{41}$. Em estudo com grupos étni- 
cos sobre fatores de risco cardiovasculares no sul da Ásia verificou-se predominância de acidente vascular cerebral em pacientes negros ${ }^{42}$.

No Brasil, as doenças cardiovasculares são a principal causa de mortalidade e comparando as taxas ajustadas por idade das principais cidades brasileiras com as de alguns países, apresentam valores elevados, sobretudo entre as mulheres ${ }^{43}$. Quanto maior o acúmulo de gordura abdominal, maior o risco de mortalidade total, pois essa distribuição abdominal está associada a maiores riscos de hipertensão, diabetes, resistência insulínica, dislipidemia, aterosclerose e doença hepática gordurosa não alcoólica ${ }^{43,44}$. Estudo prévio em comunidades quilombolas do Maranhão já demonstrou que esse perfil de morbidade por doenças cardiovasculares é o mais frequente entre idosos dessas comunidades, em ambos os sexos, porém é maior entre as mulheres ${ }^{1}$.

Neste estudo, apesar de não ter sido observado altos valores de desnutrição e nem a presença de doenças cardiovasculares, os indicadores antropométricos aqui escolhidos permitem ressaltar que a desnutrição em indivíduos com doença cardiovascular interfere nos desfechos clínicos, complicações durante a internação hospitalar, aumento das infecções, e consequente incremento do risco de morbimortalidade ${ }^{14}$. Assim, a compreensão do perfil nutricional de uma população é importante para verificar de forma precoce os fatores de risco associados, buscando o desenvolvimento de novas equações antropométricas que considerem a coexistência e sobreposição de fatores de risco ${ }^{45}$, sobretudo em populações mais vulneráveis como idosos e remanescentes de quilombos.

Entre as limitações do trabalho estão o tipo de estudo que retrata a avaliação da população em um único momento. Os entrevistadores foram treinados para realizar as medidas antropométricas, e assim, possíveis diferenças entre eles seriam residuais. Parte das medidas antropométricas utilizadas não distinguem a composição corporal. Todavia, representam medidas indispensáveis à avaliação do estado nutricional do idoso devido à fácil aplicabilidade prática e baixo custo. Medidas avaliadas neste estudo apresentam grande relação com a situação de saúde e doença dos idosos e o risco nutricional e cardiovascular observado pode decorrer também da deterioração das condições de saúde que os idosos passaram.
Deste modo, o processo de envelhecimento está relacionado com os riscos do desenvolvimento de doenças cardiovasculares, em que a situação socioeconômica, padrões de alimentação, metabolismo e condição física são algumas das características que devem ser avaliadas ${ }^{46}$.

Neste estudo, a utilização de diferentes indicadores do estado nutricional auxiliou na avaliação completa do diagnóstico nutricional e alguns deles apresentaram correlação significativa entre si. O PB é uma medida que avalia desnutrição e excesso de peso, sendo uma medida útil em situações em que não é possível mensurar peso e altura para a obtenção do IMC, por exemplo, em idosos acamados ${ }^{47}$. Já a PPE é uma das medidas mais sensíveis para mudanças de massa muscular em idosos ${ }^{14}$. Neste sentido, essa medida foi muito útil no diagnóstico de desnutrição, mas, também, como um dos indicadores de sarcopenia ${ }^{33}$ que os idosos quilombolas apresentaram.

Dessa forma, este estudo indicou que idosos quilombolas avaliados viviam em vulnerabilidade socioeconômica, com precárias condições de moradia e saneamento básico e, apresentaram alta prevalência de baixo peso, perda de massa muscular e alto risco cardiovascular. As mulheres apresentavam maiores prevalências de inadequado estado nutricional e risco cardiovascular do que os homens. Os idosos do grupo de maior idade tinham alta prevalência de desnutrição com perda de massa muscular, enquanto os mais jovens apresentavam maior prevalência de desnutrição segundo a PB. Logo, intervenções que garantam adequado estado nutricional para esses grupos são importantes e podem evitar complicações atuais e futuras, e promover a qualidade de vida e saúde dos idosos quilombolas.

\section{Colaboradores}

TC Silva participou da coleta de dados, na redação do artigo e da sua versão final. C Martins Neto participou na redação do artigo e da sua versão final. CA Carvalho, PCAF Viola, LS Rodrigues e BLCA Oliveira trabalharam em todas as etapas desde a concepção do estudo até a revisão da versão final do artigo. 


\section{Agradecimentos}

Aos idosos das comunidades quilombolas que participaram da pesquisa e as lideranças comunitárias. À Prefeitura Municipal de Bequimão, juntamente com a Secretaria de Assistência Social, às equipes da Estratégia da Família, e à UFMA pelo apoio logístico.

\section{Referências}

1. Costa ASV, Rodrigues LS, Cabral Júnior JD, Coimbra LC, Oliveira BL. Survey of the living conditions and health status of older persons living in Quilombola communities in Bequimão, Brazil: the IQUIBEQ Project. J Public Health (Berl.) 2021; 29:1061-1069.

2. Soares DA, Barreto SM. Indicadores nutricionais combinados e fatores associados em população Quilombola no Sudoeste da Bahia, Brasil. Cien Saude Colet 2015; 20(3):821-832.

3. Silveira VNC, Padilha LL, Frota MTBA. Desnutrição e fatores associados em crianças quilombolas menores de 60 meses em dois municípios do estado do Maranhão, Brasil. Cien Saude Colet 2020; 25(7):2583-2594.

4. Freitas DA, Caballero AD, Marques AS, Hernández CIV, Antunes SLNO. Saúde e comunidades quilombolas: uma revisão da literatura. Rev Cefac 2011; 13(5):937-943.

5. Andrade JS, Barroso BYC, Santos FAZ, Lima GS, Lopes TCR, Oliveira FBM. Capacity Of Self-Care In Health In The Black Population Quilombola. Rev Cien Saberes 2016; 2(4):291-296.

6. Soares DA, Kochergin CN. Fatores associados à obesidade em idosos Quilombolas, Bahia, Brasil. Rev APS 2017; 20(2):174-184

7. Vieira VK, Lazarotto AK, Antes DC, Silva JC, Motter NS, Teló VLB, Treco IC, Lucio LC. Prevalência e preditores do excesso de peso e do risco cardiovascular em mulheres quilombola de Palmas, PR. Braz J Develop 2019; 5(12):32277-32299.

8. Cunha BS, Souza CRG, Prudente LOB, Osório NB, Silva Neto LS. Sarcopenia em idosas quilombolas: Análise das variáveis antropométricas e de força de preensão manual. Rev Patol Tocantins 2017; 4(3):9-15.

9. Associação Brasileira de Empresas de Pesquisa (ABEP). Critério Brasil: Critério de Classificação Econômica Brasil 2018 [Internet]. 2016 [acessado 2019 set 12]. Disponivel em: http://www.abep.org/criterio -brasil.

10. Brasil. Ministério da Saúde (MS). Secretaria de Atenção à Saúde. Departamento de Atenção Básica. Orientações para a coleta e análise de dados antropométricos em serviços de saúde: Norma Técnica do Sistema de Vigilância Alimentar e Nutricional - SISVAN. Brasília: MS; 2011.
Este projeto foi parcialmente financiado pela Fundação de Pesquisa do Estado Maranhão (FAPEMA, Edital universal) e Conselho Nacional de Desenvolvimento Científico e Tecnológico (CNPq, Edital universal) e a Coordenação de Aperfeiçoamento de Pessoal de Nível Superior - Brasil (CAPES) - Código de Financiamento 001, ao Programa de Pós-Graduação em Saúde Coletiva.

11. World Health Organization (WHO). Physical status: the use and interpretation of anthropometry. Geneva: WHO; 1995.

12. Sampaio LR. Avaliação nutricional e envelhecimento. Rev Nutr 2004; 17(4):507-514.

13. Lohman TG, Roche AE, Martorell R. Anthropometric standardization reference manual. Illinois: Human Kinetics Book; 1988.

14. Paz RC, Silva APS, Sottomaior CLC, Gomes LF, Baptistella MKCS, Fortes RC. Sugestão de protocolo clínico para idosos cardiopatas assistidos pelo sistema único de saúde. Rev Cient Sena Aires 2018; 7(2):88-94.

15. Santos DMS, Prado BS, Oliveira CCC, Almeida-Santos MA. Prevalência da Hipertensão Arterial Sistêmica em Comunidades Quilombolas do Estado de Sergipe, Brasil. Arq Bras Cardiol 2019; 113(3):383-390.

16. Rosa DLF, Areosa SVC. Caracterização Socioeconômica De Idosos Residentes Do Meio Rural. Rev Jovens Pesqu 2019; 9(1):81-91.

17. Rocha GBF. A importância das condições socioeconômicas na elaboração de políticas públicas voltadas à saúde do idoso. Rev Longeviver 2019; 1(3):10-26.

18. Diz JBM, Queiroz BZ, Tavares LB, Pereira LSM. Prevalência de sarcopenia em idosos: resultados de estudos transversais amplos em diferentes países. Rev Bras Geriatr Gerontol 2015; 18(3):665-678.

19. Silva HP, Padez C, Moura EAF, Filgueiras LA. Obesity, hypertension, social determinants of health and the epidemiologic transition among traditional Amazonian populations. Ann Hum Biol 2016; 43(4):371-381.

20. Santos RC. Estado nutricional, anemia e fatores de risco cardiometabólico em adultos e idosos quilombolas de Goiás [tese] Goiânia: Universidade Federal de Goiás; 2016.

21. Assumpção D, Borim FSA, Francisco PMSB, Neri AL. Fatores associados ao baixo peso em idosos comunitários de sete cidades brasileiras: Estudo FIBRA. Cien Saude Colet 2018; 23(4):1143-1150.

22. Mussi RFF, Queiroz BM, Petroski EL. Excesso de peso e fatores associados em quilomboras do médio São Francisco baiano, Brasil. Cien Saude Colet 2018; 23(4):1193-1200. 
23. Venturini CD, Engroff P, Gomes I, Carli GA. Prevalência de obesidade associada à ingestão calórica, glicemia e perfil lipídico em uma amostra populacional de idosos do Sul do Brasil. Rev Bras Geriatr Gerontol 2013; 16(3):591-601.

24. Wanzeler FSC, Nogueira JAD. Atividade física em populações rurais do Brasil: uma revisão de literatura. Rev Bras Cien Mov 2019; 27(4):228-240.

25. Boscatto EC, Duarte MFS, Barbosa AR. Nível de atividade física e variáveis associadas em idosos longevos de Antônio Carlos, SC. Rev Bras Ativ Fis Saude 2012; 17(2):132-136.

26. Bezerra VM, Andrade AMS, César CC, CaiaffaII WT. Comunidades quilombolas de Vitória da Conquista, Bahia, Brasil: hipertensão arterial e fatores associados. Cad Saude Publica 2013; 29(9):1889-1902.

27. Freitas IA, Rodrigues ILA, Silva IFS, Nogueira LMV. Perfil sociodemográfico e epidemiológico de uma comunidade quilombola na Amazônia Brasileira. Rev Cuid 2018; 9(2):2187-2200.

28. Silva CRS, Maués EM, Miranda RNA, Santos TC, Carvalho EP, Serrão FO. Estado nutricional de idosos internados na clínica médica de um hospital universitário. Nutr Bras 2018; 17(3):170-177.

29. Rosa EPC, Bacalhau SPOS, Silva SA, Santos IAM, Borges FDS, Silva GA, Araújo ERS, Medeiros GC. Risco e evolução do estado nutricional de adultos e idosos hospitalizados com distúrbios neurológicos. Nutr Clín Diet Hosp 2019; 39(2):46-53.

30. Miranda RNA, Paiva MB. Antropometria e consumo alimentar: identificador do estado nutricional de idosos. Nutr Bras 2019; 18(3):141-150.

31. Miranda RA, Carvalho EP, Amorim YR, Santos KS, Serrão FO. Conhecendo a Saúde Nutricional de Idosos Atendidos em uma Organização não Governamental, Benevides/PA. Rev Conexao UEPG 2017; 13(3):512-529.

32. Almeida SS, Costa GS, Fernandes JML, Mendes LS, Fonseca RM. Indicadores socioeconômicos, sociodemográficos, saúde e nutricionais da comunidade remanescente quilombola Mangueiras. In: Ramos EMLS, Almeida SS, Araújo AR, Santos RP, Arede ANF, Lima EJS, organizadores. Métodos e ações nutricionais em quilombos. Belém: UFPA; 2016. p. 79-110.

33. Pagotto V, Santos KF, Malaquias SG, Bachion MM, Silveira EA. Circunferência da panturrilha: validação clínica para avaliação da massa muscular em idosos. Rev Bras Enferm 2018; 71(2):322-328.

34. Pimentel GMC, Silva SC. Avaliação do consumo alimentar e composição corporal entre idosos praticantes e não praticantes de exercício físico. RBNE 2019; 13(80):505-512.

35. Braga AVP, Tavares HC, Pereira Vasconcelos PA, Araujo EKR, Freitas LFF, Vieira SCR. Perfil nutricional e incidências patológicas dos idosos atendidos na clínica escola de Nutrição de Juazeiro do Norte-CE. RBONE 2019; 13(79):440-445.

36. Santos LP, Silva JMCS, Reis VMCP, Rocha JSB, Freitas RF. Nível de atividade física de idosos participantes de grupo de convivência e fatores associados. RBPFEX 2019; 13(83):459-466.

37. Freitas AP, Vogel P, Fassina P, Adami FS. Relação da qualidade de vida com o estado nutricional de idosos. Rev Bras Qual Vida 2017; 9(1):30-44.
38. Cordovil YF, Almeida SS. Variáveis antropométricas e fatores de risco cardiovasculares associados em Quilombolas Marajoaras. RBONE 2018; 12(71):406-415.

39. Barbosa MBL, Barbosa JB, Guerra LFA, Barbosa MFL, Barbosa FL, Barbosa RL, Guida DL, Martins MLB, Bouskela E, Nascimento MDSB, Melo GSO, Castro MMS. Dyslipidemia and cardiovascular risk in Afrodescendants: a study of the Quilombola communities in Maranhão, Brazil. Rev Bras Med Fam Comunidade 2015; 10(36):1-10.

40. Carnethon MR, Pu J, Howard G, Albert MA, Anderson C, Bertoni AG, Mujahid MS, Palaniappan L, Taylor Jr HÁ, Willis M, Yancy CW. Cardiovascular Health in African Americans: A Scientific Statement From the American Heart Association. Circulation 2017; 136(21):e393-e423.

41. Mensah GA, Sampson UKA, Roth GA, Forouzanfar MH, Mohsen Naghavi, Murray CJL, Moran AE, Feigin VL. Mortality from cardiovascular diseases in subSaharan Africa, 1990-2013: a systematic analysis of data from the Global Burden of Disease Study 2013 Cardiovasc J Afr 2015; 26(2):S6-S10.

42. George J, Mathur R, Shah AD, Pujades-Rodriguez M, Denaxas S, Smeeth L, Timmis A, Hemingway H. Ethnicity and the first diagnosis of a wide range of cardiovascular diseases: Associations in a linked electronic health record cohort of 1 million patients. PLoS One 2017; 12(6):e0178945.

43. Martinazzo J, Zemolin GP, Spinelli RB, Zanardo VPS, Ceni GC. Avaliação nutricional de mulheres no climatério atendidas em ambulatório de nutrição no norte do Rio Grande do Sul, Brasil. Cien Saude Colet 2013; 18(11):3349-3356.

44. Xavier LA, Souza LP, Freire GR, Mota GA, Gonçalves JTT, Rocha JSB, Baldo MP, Santo LRE. Avaliação de perfil antropométrico associado a fatores sociocomportamentais e clínicos em mulheres climatéricas. Rev Enferm Atual Derme 2020; 91(29):10-16.

45. Simões FS, Fernandes Filho J. Utilização de indicadores antropométricos de referência em idosos na avaliação dos níveis de saúde. FIEP BULLETIN 2013; 83(n. esp.):1-7.

46. Leal JB, Leal JB, Borges YL, Oliveira DNC, Cavalcante DS, Maia MAS, Moura MIL, Rocha Neta AS. Modificações na composição corporal de mulheres em risco cardiovascular pelo método pilates. RBONE 2019; 12(76):1009-1014.

47. Garcia AM, Romani SAM, Lira PIC. Indicadores antropométricos na avaliação nutricional de idosos: um estudo comparativo. Rev Nutr 2007; 20(4):371-378.

Artigo apresentado em 29/05/2020

Aprovado em 06/11/2020

Versão final apresentada em 08/11/2020

Editores-chefes: Romeu Gomes, Antônio Augusto Moura da Silva 